\title{
Modeling System-Level Dynamics of Direct XR Sessions over mmWave Links
}

\section{Citation}

Ali, A., Galinina, O. and Andreev, S., Modeling System-Level Dynamics of Direct XR Sessions over mmWave Links. In 2020 IEEE 31st Annual International Symposium on Personal, Indoor and Mobile Radio Communications (pp. 1-7). IEEE.

Year

2020

Version

Authors' camera-ready version

Link to publication

https://ieeexplore.ieee.org/document/9217119

\section{Published in}

2020 IEEE 31st Annual International Symposium on Personal, Indoor and Mobile Radio

Communications

DOI

$\underline{\text { 10.1109/PIMRC48278.2020.9217119 }}$

\section{License}

This publication is copyrighted. You may download, display, and print it for your own personal use. Commercial use is prohibited.

\section{Takedown policy}

If you believe that this document breaches copyright, please contact the authors, and we will investigate your claim.

\section{BibTex entry}

@inproceedings\{ali2020modeling, title $=\{$ Modeling System-Level Dynamics of Direct XR Sessions over mmWave Links $\}$, author $=\{$ Ali, Asad and Galinina, Olga and Andreev, Sergey $\}$,

booktitle $=\{2020$ IEEE 31st Annual International Symposium on Personal, Indoor and Mobile Radio Communications\},

pages $=\{1--7\}$,

year $=\{2020\}$,

organization $=\{$ IEEE $\}\}$ 


\title{
Modeling System-Level Dynamics of Direct XR Sessions over mmWave Links
}

\author{
Asad Ali $^{1,2}$, Olga Galinina ${ }^{1}$, and Sergey Andreev ${ }^{1}$ \\ ${ }^{1}$ Tampere University, Tampere, Finland \\ ${ }^{2}$ Brno University of Technology, Brno, Czech Republic \\ \{asad.ali, olga.galinina, sergey.andreev\}@tuni.fi
}

\begin{abstract}
To improve the quality of experience (QoE) and prolong the battery life, high-end wearable devices may offload their computations - partially or fully - to a paired computing device. One of the promising connectivity solutions, due to heavy load, is millimeter-wave (mmWave) technologies, which offer wide bandwidth and promise to provide extreme throughput and low latency. The features of the mmWave access and the use of sophisticated beamforming techniques have posed a whole new set of problem formulations related to directionality. Over the past decade, stochastic geometry has been extensively used to study directional mmWave connectivity in static deployments; however, there remains a research gap of employing directionality in highly dynamic scenarios. To bridge this gap, in this paper, we analyze the effects of mmWave directionality for non-static device-todevice (D2D) links, typical for high-end wearable applications. We propose a queueing-theoretical approach to capturing the dynamics of the representative mmWave D2D scenario and derive approximations for the key system-level metrics of interest. Our numerical results yield important insights on the role that the directivity has in changing the interference footprint in dynamic D2D systems.
\end{abstract}

\section{INTRODUCTION}

The term Extended Reality (XR) is used to unify a wide range of immersive technologies, such as Augmented Reality (AR), Virtual Reality (VR), and Mixed Reality (MR). XR devices are being envisaged as the next-generation mobile computing platforms after smartphones [1] and as a "killer application" for 5G/5G+ networks [2], [3]. However, XR is still in its nascent stages of evolution, and much remains to be done before these prospects can materialize.

Today, the booming XR market offers (i) tethered hardware connected via cables to a resource-rich computing device (PC or gaming console) and (ii) standalone devices performing all the required computations locally. The former equipment (such as Oculus Rift, HTC Vive, and Sony PlayStation VR), while providing higher graphics quality, confine XR users to limited space (e.g., a living room) proximate to the computing device and restrict their physical movement, thus hampering the immersive experience. On the other hand, the standalone XR hardware (such as Oculus Quest, Lenovo Mirage Solo, and Xiaomi Mi VR) offers more freedom at the cost of lower graphics quality due to reduced computation capabilities and heat dissipation constraints.

For a richer immersive experience, both high graphics quality and unrestricted user mobility are essential. Consequently, new wireless tethering solutions (e.g., HTC VIVE Wireless adapter and DisplayLink Wireless VR adapter) relying on high bandwidth radio links have recently appeared on the market. Albeit these options offer better quality of experience (QoE), the user is still restricted to remain in proximity of a stationary computing device.

To overcome the geographical limitations, cloud/edge computing solutions have also been proposed, where the XR device may offload intense computations to remote servers; however, with current technologies, the motion-to-photon (MTP) latency requirement of less than $20 \mathrm{~ms}$ has not been achieved [4]. In recent years, mobile edge computing (MEC) technology that allows XR users to offload their computations to the network edge has attracted significant attention but also raised a variety of privacy and security concerns [5].

Alternatively, a less explored approach is to utilize the device-to-device (D2D) paradigm to wirelessly offload computations - either in part or in full - to a personal portable device (i.e., smartphone or laptop) that has more computational resources. Facebook Technologies LLC, more popularly known as Oculus, filed a patent [6] for portable compute cases that can perform computations for an XR device and transfer the computed data back [7].

One of the attractive radio connectivity solutions for XR is the use of millimeter-wave (mmWave) technologies, which promise to enable higher throughput and lower latency but also suffer from stronger signal attenuation. To compensate for the increased path loss, mmWave devices have to rely on highly directional transmissions by employing various beamforming techniques. Lately, mmWave wireless standards, such as 5G New Radio (NR) and IEEE 802.11ad/ay, have been gaining momentum. For example, the 3GPP Rel-17 proposes the use of NR sidelink for XR D2D communication [8], [9], while alternative standards from the IEEE 802.11 family can also be utilized to establish direct connections [10].

The use of mmWave beamforming techniques has raised a whole new set of problem formulations related to directionality. Over the past decade, stochastic geometry has been used extensively to study directional mmWave access in static deployments [11], [12]. However, there remains a research gap of exploring directionality in highly dynamic scenarios, which are the key features of future XR operation. To bridge that gap, in this work, we analyze the effects of mmWave directionality for dynamic D2D links having random durations. The contribution 
of this work is two-fold: we (i) develop a queueing-theoretical approach to capturing the system dynamics in mmWave D2D scenarios, and (ii) derive approximations for the essential system-level metrics as well as provide important insights on the interference footprint in directional mmWave networks.

The remainder of this paper is organized as follows. Section II introduces the system model and our key assumptions. In Section III, we conduct the core queueing-theoretical analysis and derive the sought metrics of interest. In Section IV, we provide selected numerical results to illustrate the effects of mmWave directionality in dynamic scenarios, and, finally, draw the main conclusions in Section V.

\section{System Model AND Assumptions}

In this section, we detail our system model and outline the underlying assumptions. We address a D2D scenario that mimics a dynamic set of independent personal networks. In particular, we explore the $2 \mathrm{D}$ case to capture the features of conventional 2D beamforming procedures, which control the radiation pattern of linear antenna arrays in one plane. We consider pairs of devices located within a large area of interest; these pairs represent, e.g., XR wearables and their connected computing devices that exchange high-rate traffic. For the sake of exposition, we term one of the paired devices $a$ transmitter ( $\mathrm{Tx})$, while the other one is referred to as a receiver $(\mathrm{Rx})$. We note, however, that this notation does not restrict our methodology to uplink or downlink traffic. We assume that the locations of Tx devices follow the Poisson Point Process (PPP) and, hence, are distributed uniformly within the area of interest. For each Tx device, its paired computing device $(\mathrm{Rx})$ is located at a distance of $d_{\max }$ in a random direction. For the sake of tractability, in our analysis, we assume a fixed $d_{\text {max }}$; however, the proposed approach is also valid for random distances with the expected value of $d_{\max }$.

\section{A. Directionality Assumptions}

The data exchange between paired devices relies on highly directional transmission and omnidirectional reception [13]. The antenna radiation patterns have axial symmetry with respect to the antenna boresight, while the antenna beams are assumed to be perfectly aligned and oriented along the boresight. We employ this assumption since XR devices may utilize advanced beamforming techniques, e.g., based on compressed sensing [14], or use sufficiently narrow beams.

Further, we decompose the directivity gain into two parts: (i) the maximum directivity $D_{0}$ achieved in the direction of the antenna boresight and (ii) directivity reduction factor $\rho(\alpha)$ that decreases the antenna gain due to the angular deviation $\alpha$, i.e., the angle between the direction to the $\mathrm{Rx}$ and Tx antenna boresight. For analytical tractability, we employ the following simplified model for estimating the maximum directivity gain in the case of the half-power beamwidth (HPBW) $\theta$ :

$$
D_{0}=\frac{2}{1-\cos \frac{\theta}{2}} \text {. }
$$

The reduction factor is based on the approximation of the antenna radiation pattern in [15] and is given by

$$
\rho(\alpha)= \begin{cases}1-\frac{\alpha}{\theta}, & \alpha \leq \theta \\ 0, & \text { otherwise }\end{cases}
$$

In our scenario, the average path loss between two devices can be calculated as

$$
L(d)=C d^{\kappa}
$$

where $d$ is the distance between the devices, $\kappa$ is the propagation exponent, and $C$ is the propagation constant. For example, in the Friis transmission equation, $\kappa=2$ and $C=\frac{4 \pi f}{c}$, where $f$ is the carrier frequency and $c$ is the speed of light. Hence, the received power $P_{\mathrm{rx}}$ can be derived as

$$
P_{\mathrm{rx}}=P_{\mathrm{tx}} G_{\mathrm{tx}} G_{\mathrm{rx}} L^{-1}(d)=\frac{P_{\mathrm{tx}} D_{0} \rho(\alpha)}{C d^{\kappa}} .
$$

Here, the Tx (directional) antenna gain is $G_{\mathrm{tx}}=D_{0} \rho(\alpha)$, while the Rx antenna gain for all the devices is $G_{\mathrm{rx}}=1$. The maximum communication distance (i.e., along the antenna boresight) can be produced from (4) as

$$
R=\left(\frac{P_{\mathrm{tx}} D_{0}}{P_{\mathrm{thr}} C}\right)^{\frac{1}{\kappa}}
$$

where $P_{\text {thr }}$ is the minimum received power.

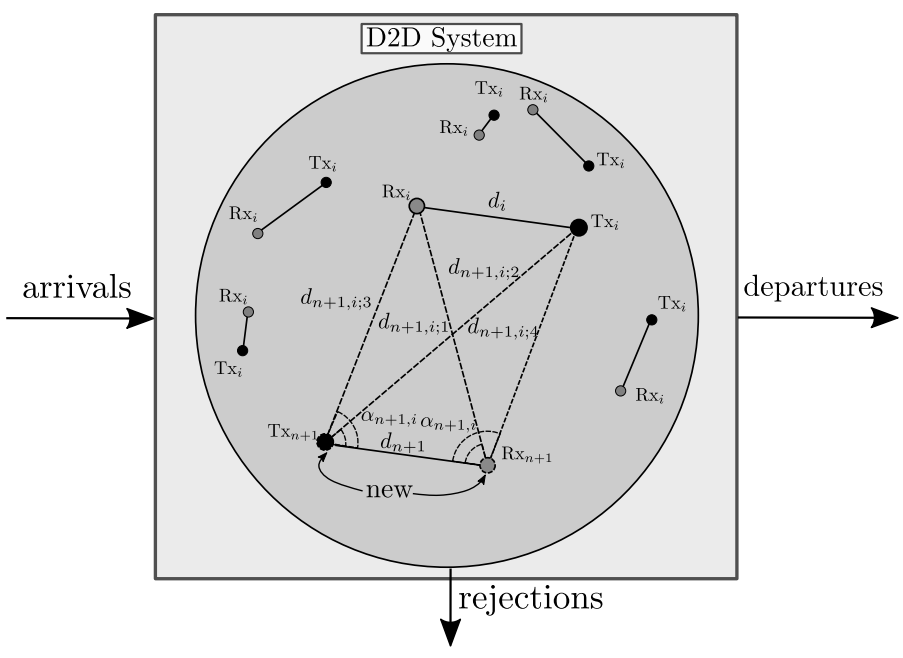

Fig. 1: Illustration of considered dynamic system.

\section{B. System Dynamics}

As we target the dynamic operation, we complement our system with queueing-theoretic assumptions to capture arrivals and departures of the device pairs in our system. Particularly, we assume that one Tx and the corresponding Rx (i.e., XR and computing devices) arrive simultaneously and immediately initiate a data transmission at the instant of time, which is regarded as the arrival of a new session. We assume that sessions arrive into the area of interest $S_{\mathrm{R}}$ according to the Poisson process with the inter-arrival time $\lambda^{-1}$, continue an 
exponential time with the average $\mu^{-1}$ if accepted, and after that leave the system permanently.

A new session $n+1$ is admitted into the system that currently serves $n$ sessions, when (i) it faces no excess interference from other devices that are currently active in the system, and (ii) it does not create excess interference for the accepted active pairs (see Fig. 1). In other words, a new session can be admitted if and only if the power of the signal received from device $X$ measured at device $Y, P_{\mathrm{rx}}^{X \rightarrow Y}$, remains below the threshold $P_{\mathrm{thr}}$. Here, $(\mathrm{X}, \mathrm{Y})$ assumes four combinations for each $i=1, . ., n$ : $\left(\mathrm{Tx}_{\mathrm{i}}, \mathrm{Rx}_{\mathrm{n}+1}\right),\left(\mathrm{Tx}_{\mathrm{i}}, \mathrm{Tx}_{\mathrm{n}+1}\right),\left(\mathrm{Rx}_{\mathrm{i}}, \mathrm{Rx}_{\mathrm{n}+1}\right)$, and $\left(\mathrm{Rx}_{\mathrm{i}}, \mathrm{Tx}_{\mathrm{n}+1}\right)$. If $P_{\mathrm{rx}}^{X \rightarrow Y}>P_{\mathrm{thr}}$ for at least one combination $(\mathrm{X}, \mathrm{Y})$, the arriving session is rejected and leaves the system before its data transmission can commence (see Fig. 1). The assumed admission control policy mimics the CSMA/CA procedures of the IEEE 802.11 systems, where a device performs channel sensing before initiating a session.

\section{Metrics of Interest}

We aim to explore the average system capacity in terms of the throughput per square meter w.r.t. the antenna directivity and the total system load per square meter. Here, the system capacity implies the number of users that can be admitted by the system simultaneously. Particularly, we calculate the expected number of active pairs and the total probability of being rejected upon arrival, referred to as the system rejection probability.

\section{AnAlysis OF System Dynamics}

In this section, we detail our approach to addressing the system dynamics in terms of the queuing theory. We derive an approximation of the queueing model that describes our system and evaluate the steady-state distribution and the core metrics of interest.

\section{A. Queueing Model}

We characterize our system by a Markov process, where states are defined by the number of active pairs and their corresponding locations. Particularly, for $n$ currently active pairs, the system state $S(t)$ may be described by a vector that includes coordinates of all $n$ pairs, $\left[n \mid\left(\omega_{1} ; v_{1}\right),\left(\omega_{2} ; v_{2}\right), \cdots,\left(\omega_{n} ; v_{n}\right)\right]$. Here, $\omega_{i}$ and $v_{i}$ describe the coordinates of Tx and Rx for the $i$-th pair. The set of all possible states of this Markov process is uncountable, and to simplify its structure, we aggregate all the states that relate to $n$ pairs into one unified state (see Fig. 2), regardless of the exact location of each device (further details about the state aggregation can be found in [16], [17]). Below, we focus on the aggregate process $\tilde{S}(t)$ and regard it as an approximation of our initial process $S(t)$.

We denote the probability that the new $(n+1)$-th arriving pair is accepted by the system given $n$ active pairs as $Q_{n+1}$. The transition from state $n$ to state $n+1$ depends on the arrival rate, and, thus, equals to $\lambda Q_{n+1}$, while the transition from state $n+1$ to state $n$ can be trivially found as $(n+1) \mu$.

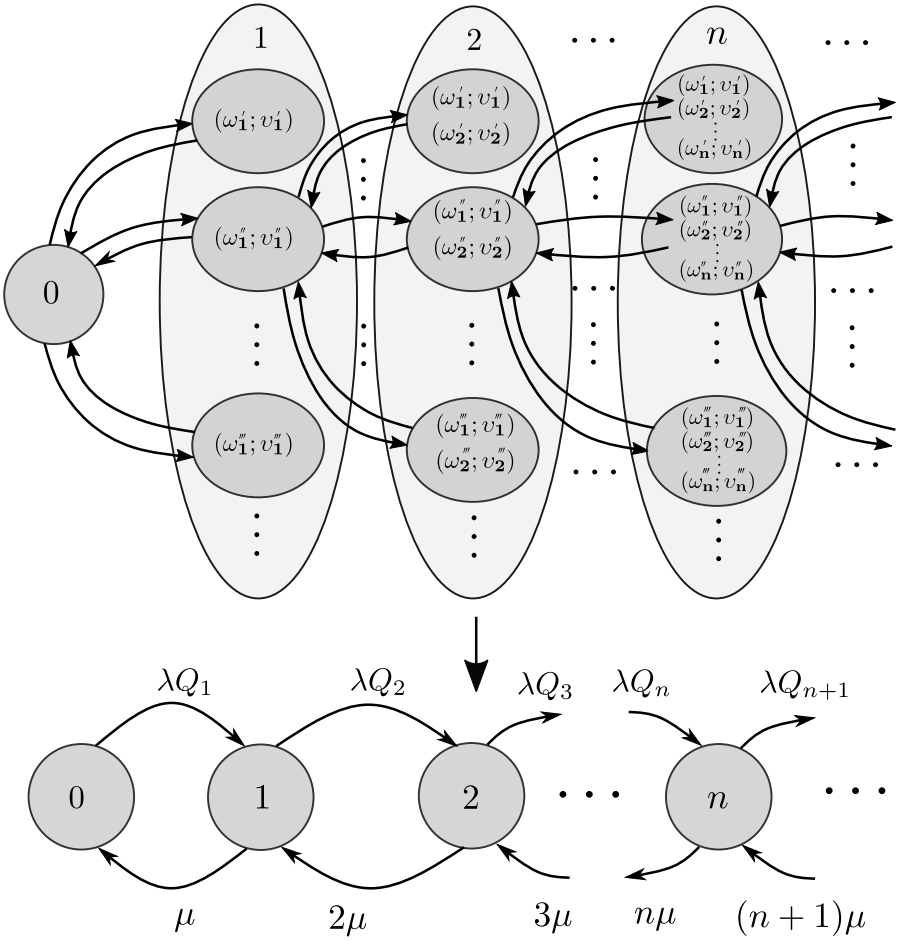

Fig. 2: Illustration of state aggregation principle.

Proposition 1. The aggregate process $\tilde{S}(t)$ is a birth-death process, and, therefore, its steady-state distribution can be calculated as

$$
\pi_{k}=\pi_{0} \frac{\lambda^{k}}{\mu^{k}} \frac{\prod_{n=1}^{k} Q_{n}}{k !},
$$

where $Q_{n}=\operatorname{Pr}\{n$-th accepted $\mid n-1$ active pairs $\}$ and $\pi_{0}=\left(\sum_{k=0}^{\infty} \frac{\lambda^{k}}{\mu^{k}} \frac{\prod_{n=1}^{k} Q_{n}}{k !}\right)^{-1}$ follows from the normalization condition.

Based on the steady-state distribution, we may obtain the metrics that characterize our system. Specifically, we target the average number of active pairs and the system rejection probability

$$
\mathrm{E}[\mathrm{N}]=\sum_{n=1}^{\infty} n \pi_{n} \text { and } \mathrm{P}_{\text {reject }}=\sum_{n=0}^{\infty}\left(1-Q_{n+1}\right) \pi_{n} .
$$

To produce the steady-state distribution and the expressions above, one requires to evaluate the probabilities $Q_{n}$. When the system is in state $n=0$, the arriving pair is accepted straightaway, which implies that $Q_{1}=1$. In the rest of this section, we focus on obtaining expressions for $Q_{n+1}, n \geq 1$.

\section{B. Expression for $Q_{2}$}

For the sake of tractability, we approximate the combined coverage area of one communicating pair with an ellipse of the size determined by the maximum communication distance and the maximum width of the beam coverage area (see Fig. 4 illustrating various HPBW values). We remind that the radiation patterns of Tx antennas of both devices in the pairs are completely identical and perfectly aligned toward 


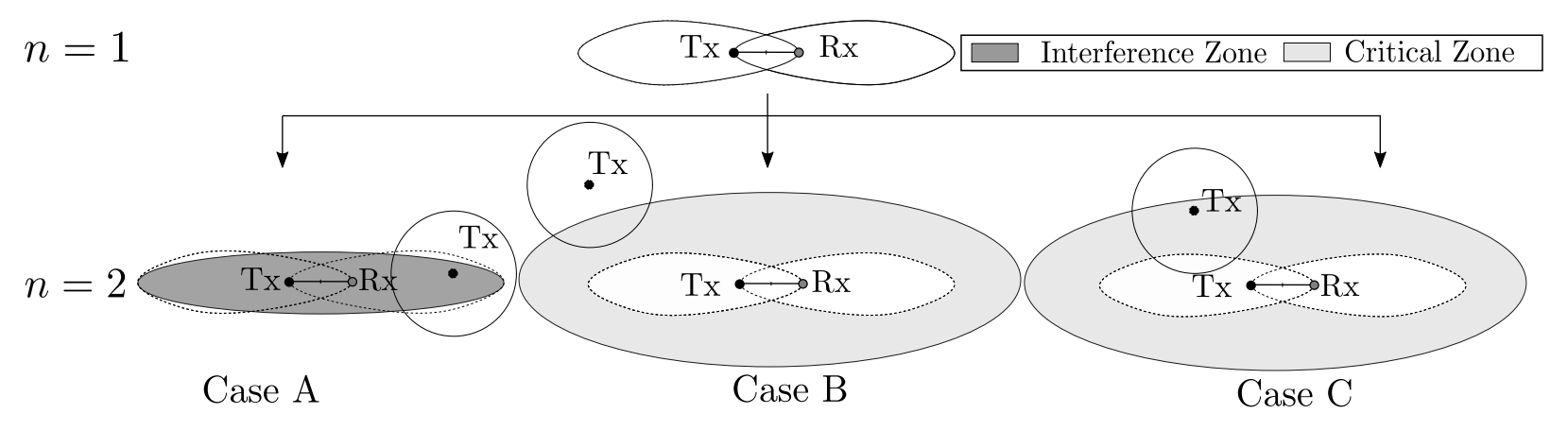

Fig. 3: Illustration of cases $\mathrm{A}, \mathrm{B}$, and $\mathrm{C}$ for $n=2$.

each other. The length of the semi-major ellipse axis equals $\left(R-\frac{d_{\max }}{2}\right)$, where $R$ is given by (5), while the length of the semi-minor axis is $x_{\max }$. The derivation of $x_{\max }$ is provided by (23) in Appendix. In what follows, we refer to the pairs of devices as to ellipses.

To determine the probability $Q_{2}$ of process $\tilde{S}(t)$, we consider the state $n=1$, where one active pair is located randomly within the area of interest. When a new ellipse arrives into the system, so that the Tx appears randomly and its Rx is located at a fixed distance, the following three cases may occur:

- Case A: the Tx falls directly into the elliptical area of the active session. We name the said elliptical area an "interference zone" since the arriving pair is being interfered by other devices. Here, the probability of the second pair (or, more generally, $(n+1)$-th pair) being accepted by the system is zero. The probability of case A to occur is geometrical and, hence, is given by $\operatorname{Pr}\{\mathrm{A}\}=\frac{A_{\mathrm{elp}}}{S_{\mathrm{R}}}$, where $S_{\mathrm{R}}$ is the area of interest and $A_{\text {elp }}=\pi\left(R-\frac{d_{\max }}{2}\right) x_{\max }$ is the area of the "interference zone". The resulting probability of case A is

$$
\operatorname{Pr}\{\mathrm{A} \mid n=1\}=\frac{\pi\left(R-\frac{d_{\max }}{2}\right) x_{\max }}{S_{\mathrm{R}}} .
$$

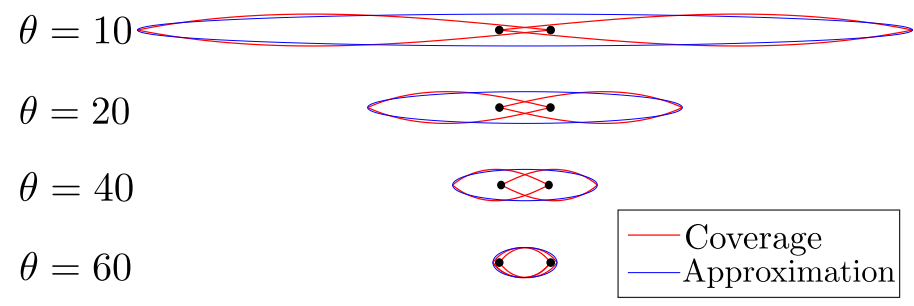

Fig. 4: Elliptical approximation of joint coverage area of D2D pair for different beamwidth $\theta$.

- Case B: the distance between the arriving Tx and the "interference zone" is greater than $d_{\max }$, and, thus, both $\mathrm{Tx}$ and $\mathrm{Rx}$ do not experience interference from the active pair. We term this extended region around the "interference zone" a "critical zone" (illustrated in Fig. 5). If the Tx device avoids the "critical zone", then the probability for this pair to be accepted is one. The probability of case $\mathrm{B}$ to occur is given as follows

$$
\operatorname{Pr}\{\mathrm{B} \mid n=1\}=\max \left(1-\frac{A_{\mathrm{crt}}}{S_{\mathrm{R}}}, 0\right),
$$

where $A_{\text {crt }}$ is the area containing the "critical zone" and the "interference zone". Therefore,

$$
\operatorname{Pr}\{\mathrm{B} \mid n=1\}=1-\frac{\pi\left(R+\frac{d_{\max }}{2}\right)\left(x_{\max }+d_{\max }\right)}{S_{\mathrm{R}}} .
$$

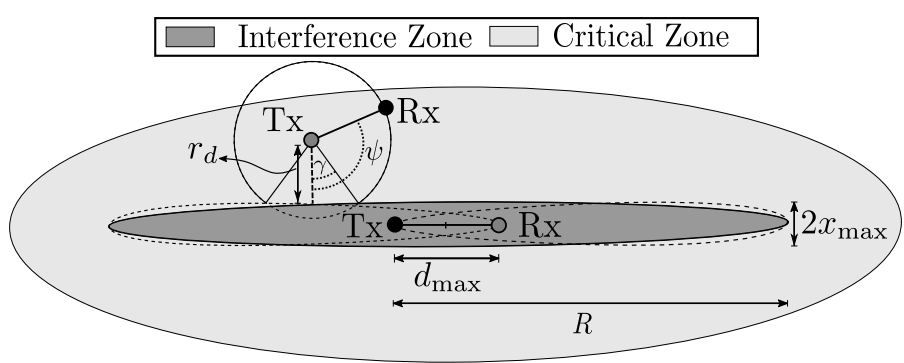

Fig. 5: Illustration of "interference zone" and "critical zone".

- Case C: the Tx is located inside the "critical zone", i.e., outside but sufficiently close to the "interference zone". Hence, there is a possibility that its Rx may fall into the elliptical area. The probability of the Tx appearing in the "critical zone" is given by the following:

$$
\begin{aligned}
\operatorname{Pr}\{\mathrm{C} \mid n=1\} & =\frac{A_{\text {crt }}-A_{\text {elp }}}{S_{\mathrm{R}}}= \\
& \frac{\pi d_{\max }\left(x_{\max }+R+\frac{d_{\max }}{2}\right)}{S_{\mathrm{R}}} .
\end{aligned}
$$

The probability of the pair being accepted in case $\mathrm{C}$ lies in the interval $(0,1)$ and is captured by Proposition 2 .

Proposition 2. The probability that a pair arriving at the state $n=1$ is accepted in case $\mathrm{C}$ is given by

$$
\operatorname{Pr}\{\text { accepted } \mid \mathrm{C}, n=1\}=1-\frac{1}{\pi} \text {. }
$$

Proof. We assume that the Tx device is located inside the "critical zone" so that the distance between the ellipse and the $\mathrm{Tx}$ is $r_{d} \leq d_{\max }$. We approximate the area, where the Tx may be located, by a rectangle (i.e., straightening the borders of both ellipses in the proximity of the Tx device). Then, since 
the locations of Tx devices follow a PPP, the distribution of $r_{d}$ may be estimated as $f_{r_{d}}\left(r_{d}\right)=\frac{1}{d_{\max }}, r_{d} \in\left[0, d_{\max }\right]$.

Let us characterize the locus of possible Rx locations (see Fig. 5). The part of the circle of radius $d_{\max }$ that falls into the ellipse area belongs to the case where the new pair cannot be accepted (the arc is highlighted by a dash curve). Then, we may obtain the probability of the pair being accepted as

$$
\begin{aligned}
& \operatorname{Pr}\{\text { accepted } \mid \mathrm{C}\}= \\
& 1-\int_{0}^{d_{\max }} \mathbb{1}_{\mathrm{A}}(2 \pi-\gamma<\psi<\gamma) f_{r_{d}}\left(r_{d}\right) f_{\psi}(\psi) d r_{d} d \psi,
\end{aligned}
$$

where $\mathbb{1}_{\mathrm{A}}(\cdot)$ is an indicator function, $\psi$ is a random direction to the $\mathrm{Rx}, \gamma$ is the maximum value of $\psi$ to keep the $\mathrm{Rx}$ inside the ellipse, and $f_{\psi}(\psi)$ is the distribution of the $\mathrm{Rx}$ directions. Then, the probability $\operatorname{Pr}\{$ accepted $\mid \mathrm{C}\}$ can be rewritten as

$\operatorname{Pr}\{$ accepted $\mid \mathrm{C}, n=1\}=1-\frac{\int_{0}^{d_{\max }} \arccos \frac{r}{d_{\max }} d r}{d_{\max } \pi}=1-\frac{1}{\pi}$.

Proposition 3. The probability $Q_{2}$ can be calculated as

$$
\begin{array}{r}
Q_{2}=\operatorname{Pr}\{n+1 \mid \mathrm{C}, n=1\} \cdot \operatorname{Pr}\{\mathrm{C} \mid n=1\}+\operatorname{Pr}\{\mathrm{B} \mid n=1\}= \\
1+\frac{\pi}{S_{\mathrm{R}}}\left(x_{\max }\left(\frac{d_{\max }}{2}-R\right)-\frac{d_{\max }}{\pi}\left(x_{\max }+d_{\max }+R\right)\right) .
\end{array}
$$

\section{Expression for $Q_{n+1}$}

Following the above approach for $Q_{2}$, we may continue by calculating the generalized expression for $Q_{n+1}, n \geq 1$. Let us consider an arbitrary state $n$, where $n$ pairs are randomly located within the area of interest so that they do not interfere. Technically, the coverage areas (ellipses) of these pairs may intersect, but for the sake of simplicity, we disregard the possibility of overlapping. Hence, we may assume that the total covered area increases $n$-fold. Similar to the previous subsection, we differentiate between three cases A, B, and C, where (i) case A corresponds to the situation where the $\mathrm{Tx}$ device falls into the combined (disconnected) coverage area that forms one "interference zone", (ii) case B addresses the farther away locations of the Tx that avoid the "interference zone" and the "critical zone", by the analogy with the previous definition, and (iii) case $\mathrm{C}$ covers the $\mathrm{Tx}$ devices that appear in the "critical zone". The probability of case B, where the pair is accepted with the probability one, can be approximated by

$$
\begin{aligned}
\operatorname{Pr}\{\mathrm{B} \mid n\}= & \max \left(1-n \frac{A_{\mathrm{crt}}}{S_{\mathrm{R}}}, 0\right)= \\
& \max \left(1-n \frac{\pi\left(R+\frac{d_{\max }}{2}\right)\left(x_{\max }+d_{\max }\right)}{S_{\mathrm{R}}}, 0\right),
\end{aligned}
$$

where $A_{\text {crt }}$ is the area containing one "critical zone" and the "interference zone". The probability of case $\mathrm{C}$ is given by

$$
\operatorname{Pr}\{\mathrm{C} \mid n\}=n \frac{A_{\mathrm{crt}}-A_{\mathrm{elp}}}{S_{\mathrm{R}}}=n \frac{\pi d_{\max }\left(x_{\max }+R+\frac{d_{\max }}{2}\right)}{S_{\mathrm{R}}} .
$$

We note that the acceptance probability for the state $n$ in case $\mathrm{C}, \operatorname{Pr}\{n+1 \mid \mathrm{C}, n\}$, equals the corresponding probability for $n=1$ and can be calculated according to (12).
Proposition 4. The probability $Q_{n+1}$ can be obtained as

$$
\begin{array}{r}
Q_{n+1}=\operatorname{Pr}\{n+1 \mid \mathrm{C}, n\} \cdot \operatorname{Pr}\{\mathrm{C} \mid n\}+\operatorname{Pr}\{\mathrm{B} \mid n\}= \\
\max \left(1+\frac{n \pi}{S_{\mathrm{R}}}\left(x_{\max }\left(\frac{d_{\max }}{2}-R\right)-\frac{d_{\max }}{\pi}\left(x_{\max }+d_{\max }+R\right)\right), 0\right) .
\end{array}
$$

Here, $d_{\max }$ may represent the expected value of random distances. Having the probabilities from (15) and (18), we can obtain the state distribution given by (6) as well as estimate the average number of sessions and the rejection probability.

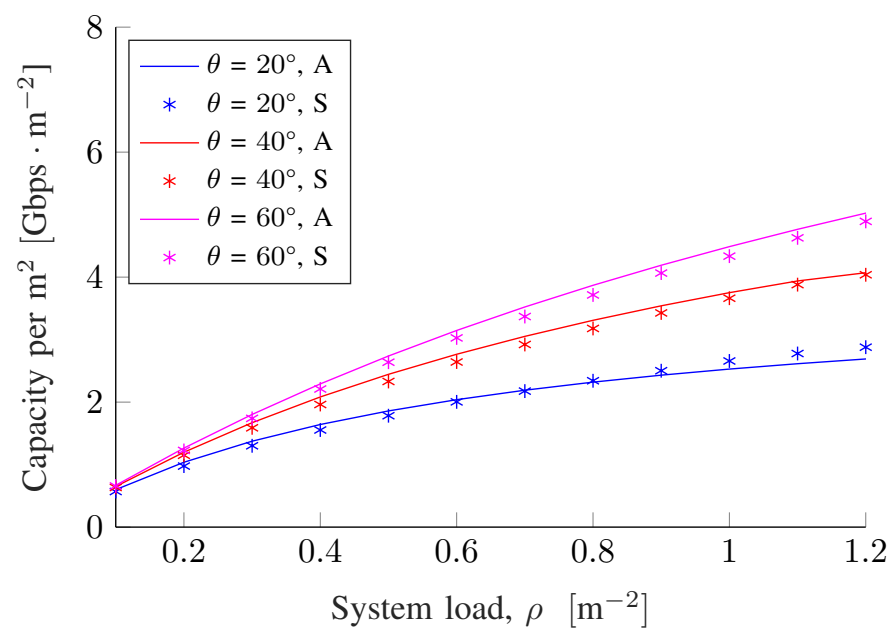

Fig. 6: System capacity per $\mathrm{m}^{2}$ vs. system load per $\mathrm{m}^{2}, \rho$, for varied beamwidth, $P_{\mathrm{tx}}=10 \mathrm{dBm}$; "A" and "S" stand for analysis and simulation results, correspondingly.

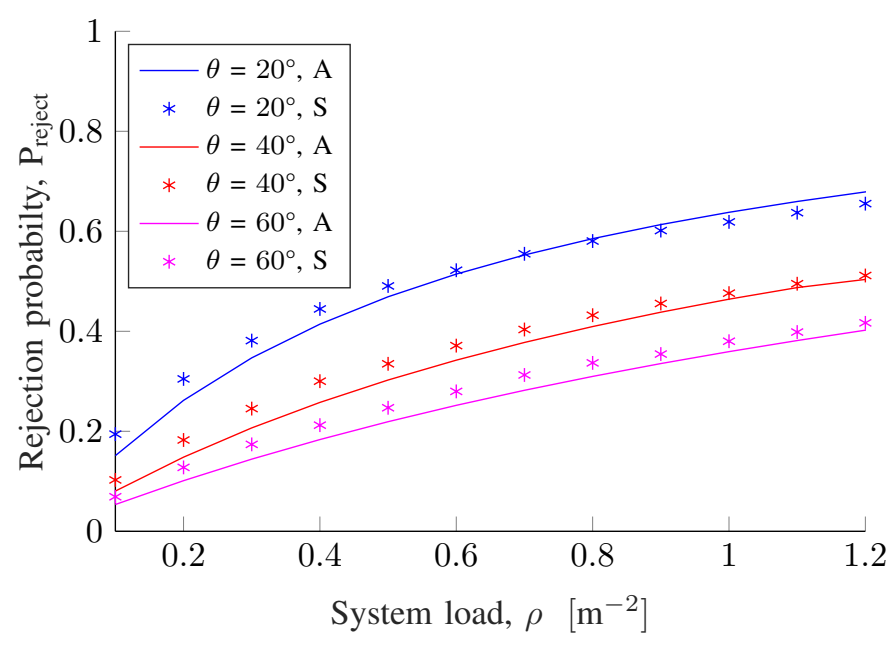

Fig. 7: Rejection probability, $\mathrm{P}_{\text {reject }}$, vs. system load per $\mathrm{m}^{2}$, $\rho$, for varied beamwidth, $P_{\mathrm{tx}}=10 \mathrm{dBm}$.

\section{NumericAl RESUlts}

In this section, we provide our selected numerical results to illustrate the effects of directional transmission in a dynamic D2D system, where pairs of devices communicate via, e.g., the IEEE 802.11ad protocol. We assume the line-of-sight 


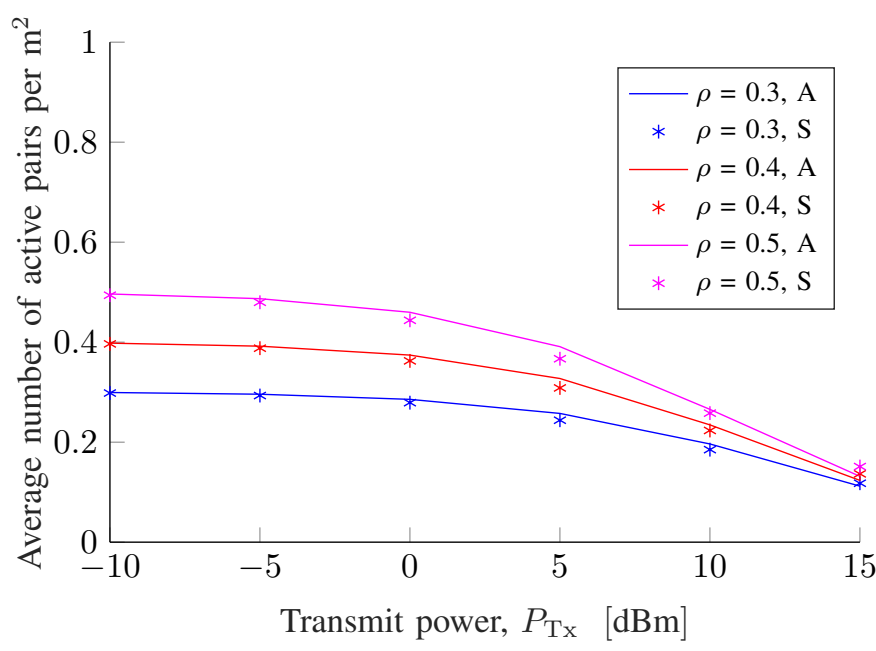

Fig. 8: Average number of pairs per $\mathrm{m}^{2}$ vs. transmit power, $P_{\mathrm{tx}}$, for varied load per unit area, $\theta=20^{\circ}$. Fewer pairs per unit area can be admitted as $P_{\mathrm{tx}}$ increases.

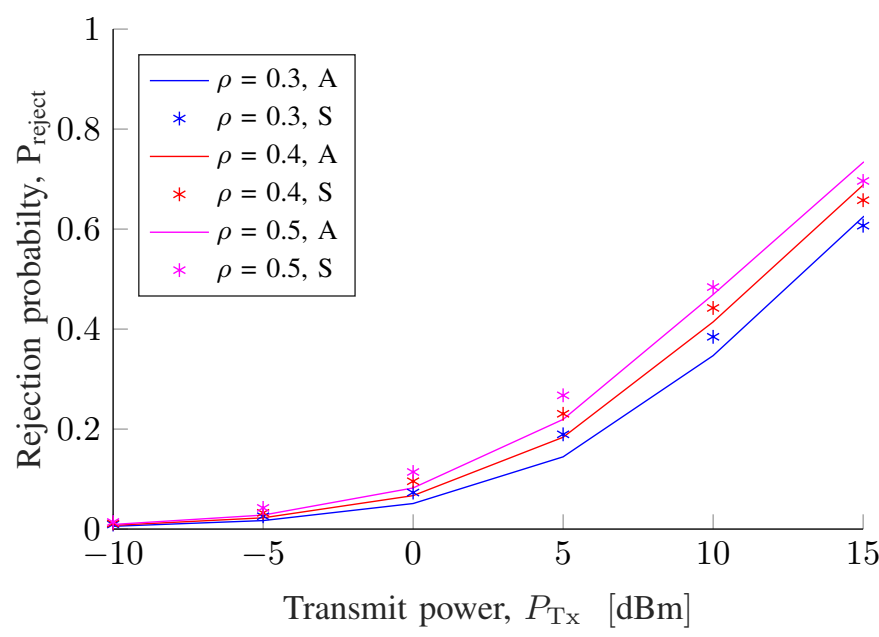

Fig. 9: Rejection probability, $\mathrm{P}_{\text {reject }}$, vs. transmit power, $P_{\mathrm{tx}}$, for varied load per unit area, $\theta=20^{\circ}$. New pairs are more likely to experience interference as $P_{\mathrm{tx}}$ increases.

TABLE I: System parameters

\begin{tabular}{|l|c|c|}
\hline \hline Description & Notation & Value \\
\hline \hline Radius of area of interest & $R_{\max }$ & $1000 \mathrm{~m}$ \\
\hline Carrier frequency & $f$ & $60 \mathrm{GHz}$ \\
\hline Bandwidth & $w$ & $2.16 \mathrm{GHz}$ \\
\hline Threshold power & $P_{\mathrm{thr}}$ & $-78 \mathrm{dBm}$ \\
\hline Transmit power & $P_{\mathrm{tx}}$ & var \\
\hline Propagation exponent & $\kappa$ & 2 \\
\hline Propagation constant & $C$ & $6.3 \times 10^{6}$ \\
\hline Beamwidth & $\theta$ & var \\
\hline Arrival rate & $\lambda$ & var \\
\hline Service rate & $\mu$ & $1 \mathrm{~s}^{-1}$ \\
\hline \hline
\end{tabular}

propagation and employ the Friis formula for calculating the path loss; however, one may consider another propagation model instead. Table I summarizes the default parameters used in this section. For the numerical results, we consider a circular area of interest $S_{\mathrm{R}}=\pi R_{\max }^{2}$.

We validate our analytical approximation by using Monte Carlo simulations. To make our model more realistic, we assume that the distance between the $\mathrm{Tx}$ and the $\mathrm{Rx}$ is random and is selected as follows. We require that paired devices are distributed in a certain $3 \mathrm{D}$ volume, which is, for simplicity, a cuboid of size $0.3 \mathrm{~m} \times 0.4 \mathrm{~m} \times 0.5 \mathrm{~m}$. To mimic the $2 \mathrm{D}$ beamforming between the devices, we consider their projections onto the $2 \mathrm{D}$ plane and further address the distance between them. In our analytical formulation, we replace $d_{\max }$ by the expected value of distances that can be obtained based on the distribution of the device locations inside a cuboid, or any other geometrical shape.

In Fig. 6, we study the dependency of the capacity per $\mathrm{m}^{2}$ on the load of paired devices per $\mathrm{m}^{2}, \rho=\frac{\lambda / \mu}{S_{\mathrm{R}}}$, for $\theta=20^{\circ}, 40^{\circ}$, and $60^{\circ}$. We assume $7 \mathrm{Gbps}$ link capacity for an active session, which corresponds to the theoretical limit of the IEEE 802.11ad protocol, and calculate the capacity per $\mathrm{m}^{2}$, accordingly. Naturally, the capacity growth gradually declines with increased load. This reveals a $\sqrt{x}$-dependency between the variables, which is a result of less favorable interference conditions and, hence, the increasing rejection probability (depicted in Fig. 7). Both plots demonstrate a tight match between the results based on analysis ("A") and simulation ("S"). A minor gap between the curves can be explained by the following two simplifications of our analytical model, where (i) "critical zones" are not allowed to overlap, and (ii) during the admission procedure, the interference check is performed only one way, in contrast to the simulations where we model full control (i.e., $n$ pairs cannot interfere with the $(n+1)$-th new pair, and vice versa). While the first simplification results in slightly overestimated rejection probability, the second one leads to "softer" admission. Depending on the system load, these opposite trends may mutually compensate to return tight upper or lower bounds on the system capacity.

Importantly, as the beam becomes narrower, the rejection probability increases. This also deteriorates the total capacity, due to the higher antenna gain, and, therefore, the expanded area of potential interference. Our results illustrate that directionality leads to a different interference footprint compared to that in the conventional microwave radio systems. This observation does not necessarily mean that the interference is negligible in highly directional systems - in fact, it increases for narrower beams as we confirm here for the case of 2D beamforming. With the 3D beamforming, we expect that the interference might decline, as the usage of, e.g., planar antenna arrays allows taking advantage of both vertical and horizontal planes. We note, however, that the system behavior in the 3D case depends on the deployment setup and needs to be studied separately.

In Fig. 8, we plot the average number of pairs, $E[N]$ per $\mathrm{m}^{2}$, versus the transmit power, $P_{\mathrm{tx}}$, for varied load per $\mathrm{m}^{2}, \rho$. The increased transmit power results in the expanded coverage area of pairs, which naturally reduces the number 
of potentially admitted links in the area. The same trend is visible in Fig. 9, where the rejection probability, $P_{\text {reject }}$, grows with $P_{\mathrm{tx}}$. Based on these results, we argue that employing mechanisms of adaptive transmit power control can be highly beneficial for reducing the interference and improving the overall performance of the mmWave-based D2D system.

\section{CONClusion}

In this paper, we evaluate the system-level dynamics of a mmWave-based D2D system that relies on the use of 2D beamforming. To assess the effects of directivity and system load, we derive a tractable analytical formulation based on queueing theoretical assumptions and a geometric approximation of the joint coverage area of paired devices. Our results display an increase in the system rejection probability and the consequent decline in its capacity in the case of narrower beamwidth or higher transmit power, as both parameters significantly affect the interference footprint. Importantly, we demonstrate that in highly directional XR networks, the interference impact is not straightforward. It depends on the type of antenna arrays and deployment and may result in deteriorated system capacity. However, the performance of a D2D system can be improved by employing appropriate schemes of adaptive power control. Potential extensions of our methodology include a consideration of 3D beamforming and power control mechanisms as well as incorporation of the human body blockage effects.

\section{APPENDIX}

\section{Calculation of $x_{\max }$}

Here, we derive the expression for a half of the maximum width of the coverage area, $x_{\max }$. We remind that at the border of the coverage area, the received power drops to its minimum level, that is, $P_{\mathrm{Rx}}=P_{\mathrm{thr}}$. From equation (4), given the deviation angle $\alpha$, one may establish the distance $d(\alpha)$ between the antenna and the border of the coverage area as

$$
d(\alpha)=\left(\frac{P_{\mathrm{Tx}} D_{0} \rho(\alpha)}{P_{\mathrm{thr}} C}\right)^{\frac{1}{\kappa}}=\left(\frac{P_{\mathrm{Tx}} D_{0}\left(1-\frac{\alpha}{\theta}\right)}{P_{\mathrm{thr}} C}\right)^{\frac{1}{\kappa}}, \text { if } \alpha \leq \theta .
$$

The $x$-coordinate of an arbitrary point at the border is

$$
x(\alpha)=d(\alpha) \sin (\alpha)=\left(\frac{P_{\mathrm{Tx}} D_{0}\left(1-\frac{\alpha}{\theta}\right)}{P_{\mathrm{thr}} C}\right)^{\frac{1}{\kappa}} \sin \alpha, \alpha \leq \theta .
$$

To maximize $x(\alpha)$, we differentiate $x$ w.r.t. $\alpha$ as

$$
\begin{aligned}
\frac{\partial x(\alpha)}{\partial \alpha} & =\frac{\partial}{\partial \alpha}\left[R\left(1-\frac{\alpha}{\theta}\right)^{\frac{1}{\kappa}} \sin \alpha\right]= \\
& =R\left(1-\frac{\alpha}{\theta}\right)^{\frac{1}{\kappa}-1}\left[\left(1-\frac{\alpha}{\theta}\right) \cos \alpha-\frac{1}{\kappa \theta} \sin \alpha\right]
\end{aligned}
$$

where $R=\left(\frac{P_{\mathrm{Tx}} D_{0}}{P_{\mathrm{thr}} C}\right)^{\frac{1}{\kappa}}$. From $\frac{\partial x(\alpha)}{\partial \alpha}=0$, it follows that

$$
\kappa(\theta-\alpha) \cos \alpha-\sin \alpha=0 \text { or } \kappa(\theta-\alpha)=\tan (\alpha) \text {. }
$$

The expression (22) is a transcendental equation w.r.t. $\alpha$ and, therefore, the optimal $\alpha^{*}$ can be found numerically. The corresponding value of $x_{\max }$ may finally be obtained as

$$
x_{\max }=x\left(\alpha^{*}\right)=d\left(\alpha^{*}\right) \sin \left(\alpha^{*}\right) .
$$

\section{ACKNOWLEDGMENT}

The authors gratefully acknowledge funding from European Union's Horizon 2020 Research and Innovation programme under the Marie Skodowska Curie grant agreement No. 813278 (project A-WEAR). The work of O. Galinina is supported by the Academy of Finland (project CROWN).

\section{REFERENCES}

[1] A. K. Noor, "Keeping it real," Mechanical Engineering, vol. 140, no. 11, pp. $40-45,2018$.

[2] M. S. Elbamby, C. Perfecto, M. Bennis, and K. Doppler, "Toward lowlatency and ultra-reliable virtual reality," IEEE Network, vol. 32, no. 2, pp. 78-84, 2018.

[3] M. Erol-Kantarci and S. Sukhmani, "Caching and computing at the edge for mobile augmented reality and virtual reality (AR/VR) in 5G," in $A d$ Hoc Networks, pp. 169-177, Springer, 2018.

[4] L. Han, S. Appleby, and K. Smith, "Problem statement: Transport support for augmented and virtual reality applications," Working Draft, IETF Secretariat, Internet-Draft draft-haniccrg-arvr-transport-problemO0, March, 2017.

[5] R. Roman, J. Lopez, and M. Mambo, "Mobile edge computing, fog et al.: A survey and analysis of security threats and challenges," Future Generation Computer Systems, vol. 78, pp. 680-698, 2018.

[6] D. Tao, D. Yang, and S. Monga, "Portable compute case for eyewear devices," Sept. 5 2019. US Patent App. 15/910,406.

[7] B. Shi, J. Yang, Z. Huang, and P. Hui, "Offloading guidelines for augmented reality applications on wearable devices," in Proceedings of the 23rd ACM international conference on Multimedia, pp. 1271-1274, 2015.

[8] 5G Americas, "5G Services Innovation - 5G Americas White Paper," tech. rep., nov 2019.

[9] A. Ghosh, A. Maeder, M. Baker, and D. Chandramouli, "5G evolution: A view on 5G cellular technology beyond 3GPP Release 15," IEEE Access, vol. 7, pp. 127639-127651, 2019.

[10] E. M. Mohamed, M. A. Abdelghany, and M. Zareei, "An efficient paradigm for multiband WiGig D2D networks," IEEE Access, vol. 7, pp. 70032-70045, 2019.

[11] K. Venugopal, M. C. Valenti, and R. W. Heath, "Interference in finitesized highly dense millimeter wave networks," in 2015 Information Theory and Applications Workshop (ITA), pp. 175-180, IEEE, 2015.

[12] K. Venugopal, M. C. Valenti, and R. W. Heath, "Device-to-device millimeter wave communications: Interference, coverage, rate, and finite topologies," IEEE Transactions on Wireless Communications, vol. 15, no. 9, pp. 6175-6188, 2016.

[13] G. Sanfilippo, O. Galinina, S. Andreev, S. Pizzi, and G. Araniti, "A concise review of $5 \mathrm{G}$ new radio capabilities for directional access at mmWave frequencies," in Internet of Things, Smart Spaces, and Next Generation Networks and Systems, pp. 340-354, Springer, 2018.

[14] N. J. Myers, A. Mezghani, and R. W. Heath, "Swift-Link: A compressive beam alignment algorithm for practical mmWave radios," IEEE Transactions on Signal Processing, vol. 67, no. 4, pp. 1104-1119, 2018.

[15] O. Galinina, A. Pyattaev, K. Johnsson, S. Andreev, and Y. Koucheryavy, "Analyzing effects of directional deafness on mmWave channel access in unlicensed bands," in 2017 IEEE Globecom Workshops (GC Wkshps), pp. 1-7, IEEE, 2017.

[16] S. Andreev, O. Galinina, A. Pyattaev, K. Johnsson, and Y. Koucheryavy, "Analyzing assisted offloading of cellular user sessions onto D2D links in unlicensed bands," IEEE Journal on Selected Areas in Communications, vol. 33, no. 1, pp. 67-80, 2014.

[17] O. Galinina, S. Andreev, M. Gerasimenko, Y. Koucheryavy, N. Himayat, S.-p. Yeh, and S. Talwar, "Capturing spatial randomness of heterogeneous cellular/WLAN deployments with dynamic traffic," IEEE Journal on Selected Areas in Communications, vol. 32, no. 6, pp. 1083-1099, 2014. 